\title{
Reliability of analysis of the bone mineral density of the second and fifth metatarsals using dual-energy $x$-ray absorptiometry (DXA)
}

N. Stewart Pritchard ${ }^{\text {, J James M. Smoliga }}{ }^{1}$, Anh-Dung Nguyen ${ }^{2}$, Micah C. Branscomb ${ }^{1}$, David R. Sinacore ${ }^{3}$, Jeffrey B. Taylor ${ }^{1}$ and Kevin R. Ford ${ }^{1 *}$

\begin{abstract}
Background: Metatarsal fractures, especially of the fifth metatarsal, are common injuries of the foot in a young athletic population, but the risk factors for this injury are not well understood. Dual-energy x-ray absorptiometry (DXA) provides reliable measures of regional bone mineral density to predict fracture risk in the hip and lumbar spine. Recently, sub-regional metatarsal reliability was established in fresh cadaveric specimens and associated with ultimate fracture force. The purpose of this study was to assess the reliability of DXA bone mineral density measurements of sub-regions of the second and fifth metatarsals in a young, active population.

Methods: Thirty two recreationally active individuals participated in the study, and the bone density of the second (2MT) and fifth (5MT) metatarsals of each subject was measured using a Hologic QDR $x$-ray bone densitometer. Scans were analyzed separately by two raters, and regional bone mineral density, bone mineral content, and area measurements were calculated for the proximal, shaft, and distal regions of the bone. Intra-rater, inter-rater, and scan-rescan reliability were then determined for each region.

Results: Proximal and shaft bone mineral density measurements of the second and fifth metatarsal were reliable. ICC's were variable across regions and metatarsals, with the distal region being the poorest.

Conclusions: Bone mineral density measurements of the metatarsals may be a better indicator of fracture risk of the metatarsals than whole body measurements. A reliable method for measuring the regional bone mineral densities of the metatarsals was found. However, inter-rater reliability and scan-rescan reliability for the distal regions were poor. Future research should examine the relationship between DXA bone mineral density measurements and fracture risk at the metatarsals.
\end{abstract}

Keywords: Stress fracture, Bone health, Bone mineral density, Dual x-ray absorptiometry, Foot, Metatarsals

\section{Background}

Metatarsal fractures are common injuries to the foot during sport participation [1]. The distribution of injuries across the metatarsals can vary within different populations. However, many epidemiological studies do not discriminate between metatarsal fractures and foot injuries, with most focusing solely on one specific metatarsal. Healing times for these injuries can

\footnotetext{
* Correspondence: kford@highpoint.edu

'Department of Physical Therapy, High Point University, High Point, NC, USA

Full list of author information is available at the end of the article
}

range from 3 to 20 weeks and may require operative treatment depending on the type and location of the fracture [2-4]. Fractures that occur near the proximal (base) of the bone can vary in prevalence and clinical impact from fractures that occur in the shaft region of the bone. Distal fractures of the second metatarsal are more common than proximal; however, proximal fractures more commonly have delayed unions and extended healing times compared with non-proximal fractures, magnifying their clinical impact [5]. 
Similar to the second metatarsal, fractures to the proximal fifth metatarsal can also have an increased impact on individuals, especially athletes [6]. Additionally, most studies support that fifth metatarsal fractures are the most common metatarsal fracture within the general population [7]. Dameron, Lawrence, and Quill classified these fractures into three anatomically separate zones. Zone 1 fractures are tuberosity avulsion fractures. Zone 2 fractures occur at the metaphyseal/diaphyseal junction and zone 3 fractures occur along the proximal diaphysis [8]. Fractures occurring in zone 2 and zone 3 can have healing times of up to 20 weeks and require operative treatment $[4,8]$. As a result, athletes may be forced to miss half, or even a whole season of competition after experiencing a proximal fifth metatarsal fracture [6].

Understanding the factors that predispose athletes to metatarsal fractures is important prior to designing injury prevention interventions. Dual-energy X-ray absorptiometry (DXA) is a radiological technique that uses $\mathrm{X}$-rays to detect body composition by utilizing the variability of mass attenuation coefficients across different types of body tissues and has been found to be reliable in determining bone mineral densities of the femoral neck and distal thigh [9]. Furthermore, it has been found that extreme deficits in bone mineral density (BMD) at the hip are associated with metatarsal fractures [10]. However, this relationship may not be as strong in athletic populations, thus a more site-specific approach may be necessary [11].

Recently, a novel method of measuring the bone mineral density of ex-vivo metatarsals was created and found to be reliable using DXA technology. This method was adapted for the total BMD of in vivo metatarsals and found to be reliable [12]. It is uncertain how further region of interest (ROI) divisions such as proximal, shaft, and distal sections affect these results. Therefore, the purpose of this study was to identify the reliability of a novel DXA analysis procedure on fifth metatarsal and second metatarsal segments in vivo. We hypothesized that within rater, between rater, and between day reliability would be reliable for all segments.

\section{Methods}

Thirty-two recreationally active individuals participated in the study (20 (62.5\%) male participants and 12 (37.5\%) female participants; age $23.5 \pm 5.9$ yrs.; mass $71.6 \pm 12.9 \mathrm{~kg}$; height $174.9 \pm 10.4 \mathrm{~cm}$ ). Sample size was based on the International Society for Clinical Densitometry's recommendations for precision assessments [13]. Participants signed a consent form to participate in the research study that was approved by High Point University's IRB. Participants presented to the laboratory on two occasions separated by at least $24 \mathrm{~h}(5.53 \pm 8.34$ days $)$. All measurements described below were conducted on each of the two occasions that participants presented to the laboratory, so that scan-rescan reliability could be computed. While both left foot and right foot were collected as described below, only the left foot was chosen to analyze for scan-rescan reliability.

A Hologic QDR x-ray bone densitometer was used to measure the bone density of the second (2MT) and fifth (5MT) metatarsals of each subject. Before scanning, a quality control procedure was performed each day using the Hologic DXA quality control phantom. The 2MT was scanned first followed by the 5MT. The foot was positioned along the length of the table on a $6 \mathrm{~cm}$ thick mat that was centered in the middle of the DXA table. Participants were instructed to sit upright with their feet flat and hip width apart. Crosshairs from the scanner allowed the position of the scanner to be adjusted and placed directly above the subject's foot which remained still during the scans. For scanning the $2 \mathrm{MT}$, the antero-posterior crosshair was positioned between the first and second metatarsals while the medio-lateral crosshair was positioned in front of the distal portion of the hallux. For scanning the $5 \mathrm{MT}$, the antero-posterior axis was positioned between the fourth and fifth metatarsals while the medio-lateral axis was positioned in front of the distal portion of the hallux. A folded cloth was placed under the lateral side of the foot to optimally position the fourth and fifth metatarsals in the scanned image.

Scans were analyzed using the Hologic lumbar spine analysis software, version 4.0 [14]. To establish interrater reliability, two trained raters followed a standardized step-by-step process to analyze the scans in a randomized order. Raters were investigators who had previous experience in full body and metatarsal DXA scan procedures. Raters were blinded and did not have access to previous scans. Once the respective scan had been selected, the rater began the analysis by adjusting the global ROI so that it encompassed the entire image $(126 \times 201$ pixels) (Fig. 1). The rater then selected bone map and deleted all bone present. The scan was then zoomed in to $150 \%$ in order to visualize the outline of the bone more clearly. The bone was outlined by moving the curser along the edge of the bone and on the points where there was the greatest amount of change in contrast. The outline was adjusted for any errors during tracing and then filled using the "fill holes" feature. Using the vertebral lines feature, the bone was then split into proximal, shaft, and distal components. Lines were made perpendicular to the ROI and were placed on the thinnest region of cortical bone on the proximal and distal ends. The software was then able to calculate the bone mineral content (BMC), area for the proximal, shaft, and distal regions. The $\mathrm{BMD}$ was then calculated as the ratio of $\mathrm{BMC}$ to area in each region.

To establish intra-rater reliability, one of the raters performed the entire analysis procedure described 


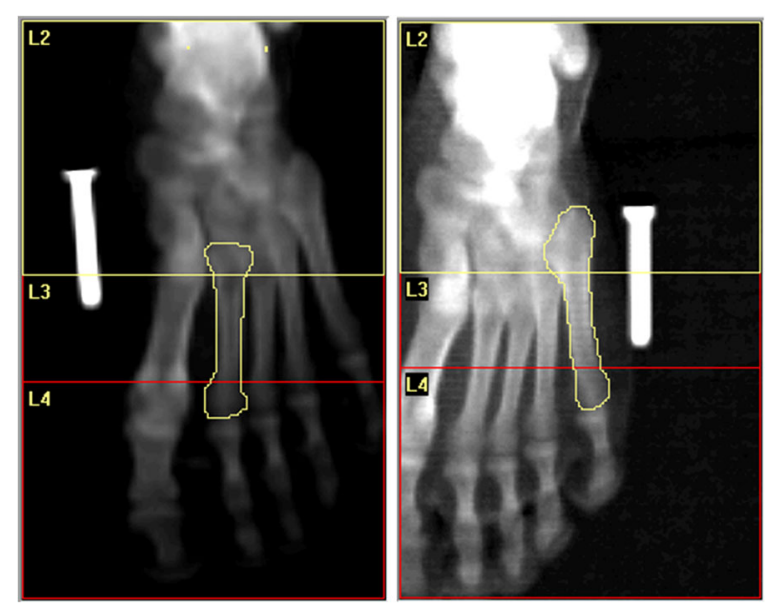

Fig. 1 Representative example of DXA analysis of the regions of interest from the lateral and medial scans. The outlined area within L2 was considered proximal. The outlined area within $\mathrm{L} 3$ was considered the shaft. The outlined area within $L 4$ was considered distal

above on identical scans twice, and these repeated analyses were done on separate days, at least a week apart. Scans from all 32 participants' first laboratory visit were used for the intra-rater reliability procedures, and assessment was performed for both $2 \mathrm{MT}$ and 5MT scans. All analyses were performed in a randomized order via a random number generator, in which scans from $2 \mathrm{MT}$ and $5 \mathrm{MT}$ were intermixed; the order was different for the first and second analysis of these identical scans.

Three different types of reliability analyses were performed: 1) intra-rater; 2) inter-rater; and 3) scanrescan. For each of these three types of analysis, single measures intraclass correlation coefficients (ICC) using the two way random effects models (corresponding to $\mathrm{ICC}_{2,1}$ terminology) were computed for each region (proximal, shaft, and distal) of $2 \mathrm{MT}$ and 5MT. 95\% confidence intervals were computed for each ICC. Excellent ICC's were considered $>85 \%$, fair ICC's were considered $>70 \%$ and poor ICC's were considered $<70 \%$. Intra-rater reliability for each region of each metatarsal was computed using the data obtained from repeated measurements by one rater (Rater 1) of identical scans, as described above. Inter-rater reliability for each region of each metatarsal was computed using data obtained from measurements by the two different raters (Rater 1 and Rater 2) from identical scans. Scan-rescan reliability for each region of each metatarsal was computed using data obtained from one rater (Rater 1) from two different scans (collected during each of the two laboratory visits). The standard error of measurement (SEM) was computed for each region for each metatarsal [15], which was normalized to the mean of each respective parameter in order to be expressed as a percentage. Statistical analyses were performed in SPSS v23.0 and Microsoft Excel.

\section{Results}

Tables 1, 2, 3, 4, 5, 6, 7, 8 and 9 present the intrarater reliability, inter-rater reliability, and scan-rescan reliability, respectively, of the proximal, shaft, distal and total regions of the $2 \mathrm{MT}$ and $5 \mathrm{MT}$. Intra-rater reliability and inter-rater reliability ranged from fair to excellent for proximal, shaft, distal, and total regions of the $2 \mathrm{MT}$ and $5 \mathrm{MT}$. The inter-rater ICC for all BMD regions of the 5MT ranged from 0.77-0.86 with SEM range of $10.2-13.7 \%$. The intra-rater ICC for all BMD regions of the 5MT ranged from 0.830.86 with SEM range of 10.1-12.0\%. The inter-rater ICC for all BMD regions of the 2MT ranged from 0.95-0.97 with SEM range of $2.8-4.6 \%$. The intrarater ICC for all BMD regions of the 2MT ranged from 0.95-0.97 with SEM range of 2.4-4.9\%. Scanrescan reliability for the proximal and shaft segments of the 2MT and the 5MT was fair. Scan-rescan ICC's of the 5MT proximal and shaft regions were 0.83 , SEM $11.0 \%$, and 0.84 , SEM 9.5\%, respectively; the ICC of the $2 \mathrm{MT}$ proximal and shaft regions were 0.77 , SEM $10.1 \%$ and 0.76 , SEM $8.4 \%$, respectively. Interestingly, scan-rescan reliability of the distal segments of the $2 \mathrm{MT}$ and $5 \mathrm{MT}$ was poor. The scanrescan ICC for the distal region of 5MT was 0.57 with an SEM of $16.2 \%$, with all other ICC $\geq 0.70$ and $\mathrm{SEM} \leq 13.7 \%$.

\section{Discussion}

Our assessment of bone mineral density was reliable for the 2MT and 5MT total ROI's. These results were consistent with the findings of other research, which also found good reliability in the 2MT and

Table 1 BMD Intra-rater reliability

\begin{tabular}{lllll}
\hline Variable & $\begin{array}{c}\text { Mean } \\
\left(\mathrm{g} / \mathrm{cm}^{2}\right)\end{array}$ & $\begin{array}{l}\text { Mean } \\
\left(\mathrm{g} / \mathrm{cm}^{2}\right)\end{array}$ & ICC $(95 \% \mathrm{Cl})$ & SEM (\%) \\
\hline $\begin{array}{l}\text { Fifth Metatarsal bone } \\
\text { Proximal }\end{array}$ & $0.5 \pm 0.11$ & $0.48 \pm 0.13$ & $0.84(0.69-0.92)$ & 10.7 \\
Shaft & $0.45 \pm 0.10$ & $0.44 \pm 0.12$ & $0.86(0.73-0.93)$ & 10.1 \\
Distal & $0.25 \pm 0.06$ & $0.25 \pm 0.07$ & $0.83(0.67-0.92)$ & 12.0 \\
Total & $0.44 \pm 0.10$ & $0.43 \pm 0.12$ & $0.86(0.72-0.93)$ & 10.1 \\
Second Metatarsal bone & & & 2.9 \\
Proximal & $0.65 \pm 0.13$ & $0.64 \pm 0.14$ & $0.95(0.90-0.98)$ & 4.9 \\
Shaft & $0.51 \pm 0.09$ & $0.51 \pm 0.09$ & $0.98(0.96-0.99)$ & 2.4 \\
Distal & $0.31 \pm 0.06$ & $0.31 \pm 0.06$ & $0.97(0.93-0.98)$ & 3.9 \\
Total & $0.49 \pm 0.08$ & $0.48 \pm 0.09$ & $0.97(0.92-0.98)$ & 3.4
\end{tabular}

Intraclass Correlation Coefficient (ICC), Standard Error of Measure (SEM) 
Table 2 BMD Inter-rater reliability

\begin{tabular}{|c|c|c|c|c|}
\hline Variable & $\begin{array}{l}\text { Mean }_{\mathrm{A} 1} \\
\left(\mathrm{~g} / \mathrm{cm}^{2}\right)\end{array}$ & $\begin{array}{l}\text { Mean }_{A 2} \\
\left(\mathrm{~g} / \mathrm{cm}^{2}\right)\end{array}$ & ICC (95\% Cl) & SEM (\%) \\
\hline \multicolumn{5}{|c|}{ Fifth Metatarsal bone } \\
\hline Proximal & $0.5 \pm 0.11$ & $0.46 \pm 0.13$ & $0.81(0.58-0.91)$ & 11.7 \\
\hline Shaft & $0.45 \pm 0.10$ & $0.45 \pm 0.12$ & $0.86(0.73-0.93)$ & 10.2 \\
\hline Distal & $0.25 \pm 0.06$ & $0.24 \pm 0.07$ & $0.77(0.57-0.89)$ & 13.7 \\
\hline Total & $0.44 \pm 0.10$ & $0.42 \pm 0.11$ & $0.83(0.64-0.92)$ & 11.0 \\
\hline \multicolumn{5}{|c|}{ Second Metatarsal bone } \\
\hline Proximal & $0.65 \pm 0.13$ & $0.64 \pm 0.14$ & $0.95(0.91-0.98)$ & 4.6 \\
\hline Shaft & $0.51 \pm 0.09$ & $0.50 \pm 0.09$ & $0.97(0.89-0.99)$ & 2.8 \\
\hline Distal & $0.31 \pm 0.06$ & $0.30 \pm 0.06$ & $0.96(0.82-0.99)$ & 4.2 \\
\hline Total & $0.49 \pm 0.08$ & $0.47 \pm 0.08$ & $0.95(0.77-0.98)$ & 3.9 \\
\hline
\end{tabular}

Intraclass Correlation Coefficient (ICC), Standard Error of Measure (SEM)

5MT [12, 14]. In addition to the total ROI, this study found good reliability of the proximal and shaft ROI's and poor reliability of the distal ROI. The poor results of the distal ROI may have to do with the quality of the DXA scans in certain regions.

Interestingly, inter-rater reliability for the distal $5 \mathrm{MT}$ and proximal $2 \mathrm{MT}$ area were not reliable. The variability within the geometry of these segments across individuals and the human error introduced by outlining the bones during analysis may have played a role in these findings [14]. Intra-rater reliability for the area of the distal 5MT and proximal 2MT were considered fair and excellent, respectively. However, inter-rater reliability and scan-rescan reliability for the distal region were poor. This suggests that while an individual rater may analyze an individual scan reliably, there were both ambiguities in the analysis procedures across raters and variability within the DXA scanning software that led to

Table 3 BMD Scan-Rescan reliability

\begin{tabular}{|c|c|c|c|c|}
\hline Variable & $\begin{array}{l}\text { Mean } 1 \\
\left(\mathrm{~g} / \mathrm{cm}^{2}\right) \\
\end{array}$ & $\begin{array}{l}\text { Mean }{ }_{D 2} \\
\left(\mathrm{~g} / \mathrm{cm}^{2}\right)\end{array}$ & ICC (95\% Cl) & SEM (\%) \\
\hline \multicolumn{5}{|c|}{ Fifth Metatarsal bone } \\
\hline Proximal & $0.5 \pm 0.11$ & $0.51 \pm 0.13$ & $0.83(0.67-0.92)$ & 11.0 \\
\hline Shaft & $0.45 \pm 0.10$ & $0.46 \pm 0.11$ & $0.84(0.68-0.92)$ & 9.5 \\
\hline Distal & $0.25 \pm 0.06$ & $0.26 \pm 0.06$ & $0.57(0.26-0.77)$ & 16.2 \\
\hline Total & $0.44 \pm 0.10$ & $0.45 \pm 0.11$ & $0.82(0.66-0.91)$ & 10.4 \\
\hline \multicolumn{5}{|c|}{ Second Metatarsal bone } \\
\hline Proximal & $0.65 \pm 0.13$ & $0.65 \pm 0.14$ & $0.77(0.58-0.89)$ & 10.1 \\
\hline Shaft & $0.51 \pm 0.09$ & $0.50 \pm 0.08$ & $0.76(0.56-0.88)$ & 8.4 \\
\hline Distal & $0.31 \pm 0.06$ & $0.30 \pm 0.07$ & $0.56(0.26-0.77)$ & 14.5 \\
\hline Total & $0.49 \pm 0.08$ & $0.48 \pm 0.09$ & $0.70(0.45-0.84)$ & 9.7 \\
\hline
\end{tabular}

Intra class Correlation Coefficient (ICC), Standard Error of Measure (SEM)
Table 4 BMC Intra-rater reliability

\begin{tabular}{|c|c|c|c|c|}
\hline Variable & $\begin{array}{l}\text { Mean }_{\mathrm{A} 1} \\
\left(\mathrm{~g} / \mathrm{cm}^{2}\right) \\
\end{array}$ & $\begin{array}{l}M_{e a n_{A 2}} \\
\left(\mathrm{~g} / \mathrm{cm}^{2}\right)\end{array}$ & ICC (95\% Cl) & SEM (\%) \\
\hline \multicolumn{5}{|c|}{ Fifth Metatarsal bone } \\
\hline Proximal & $1.87 \pm 0.63$ & $1.76 \pm 0.66$ & $0.91(0.79-0.96)$ & 11.0 \\
\hline Shaft & $1.32 \pm 0.40$ & $1.31 \pm 0.44$ & $0.90(0.81-0.95)$ & 10.4 \\
\hline Distal & $0.33 \pm 0.11$ & $0.31 \pm 0.12$ & $0.88(0.73-0.95)$ & 12.7 \\
\hline Total & $3.53 \pm 1.08$ & $3.38 \pm 1.16$ & $0.92(0.83-0.96)$ & 9.5 \\
\hline \multicolumn{5}{|c|}{ Second Metatarsal bone } \\
\hline Proximal & $1.11 \pm 0.39$ & $0.91 \pm 0.34$ & $0.65(0.21-0.84)$ & 23.0 \\
\hline Shaft & $1.68 \pm 0.46$ & $1.67 \pm 0.51$ & $0.96(0.92-0.98)$ & 5.8 \\
\hline Distal & $0.55 \pm 0.21$ & $0.54 \pm 0.19$ & $0.94(0.87-0.97)$ & 9.8 \\
\hline Total & $3.34 \pm 0.89$ & $3.12 \pm 0.90$ & $0.92(0.71-0.97)$ & 7.9 \\
\hline
\end{tabular}

Intra class Correlation Coefficient (ICC), Standard Error of Measure (SEM)

unreliable scan-rescan and inter-rater results. Standardizing the sagittal plane orientation of the ankle may help improve the reliability of these measures. Future research should attempt to further standardize these testing protocols and validate these findings with other methods such as quantitative computed tomography $(\mathrm{qCT})$ in order to improve the inter-rater and scanrescan reliability [16].

Currently, whole body or femoral neck BMD measurements are used to assess an individual's risk for stress fractures. However, the relationship between whole body bone mineral density and fractures may not be as strong in certain athletic populations. A recent study examined the relationship between bone stress injuries and the risk factors in the female athlete triad: low energy availability, menstrual dysfunction, and low total BMD and found that high risk athletes were four times more likely to experience a bone stress injury (BSI) compared to the low risk

Table 5 BMC Inter-rater reliability

\begin{tabular}{lcllc}
\hline Variable & $\begin{array}{l}\text { Mean } \\
\left(\mathrm{g} / \mathrm{cm}^{2}\right)\end{array}$ & $\begin{array}{l}\text { Mean }_{\mathrm{A2}} \\
\left(\mathrm{g} / \mathrm{cm}^{2}\right)\end{array}$ & ICC (95\% Cl) & SEM (\%) \\
\hline Fifth Metatarsal bone & & & \\
Proximal & $1.87 \pm 0.63$ & $1.66 \pm 0.64$ & $0.87(0.53-0.95)$ & 11.7 \\
Shaft & $1.32 \pm 0.40$ & $1.52 \pm 0.48$ & $0.79(0.33-0.92)$ & 10.2 \\
Distal & $0.33 \pm 0.11$ & $0.36 \pm 0.14$ & $0.85(0.69-0.93)$ & 13.7 \\
Total & $3.53 \pm 1.08$ & $3.54 \pm 1.20$ & $0.92(0.83-0.96)$ & 11.0 \\
Second Metatarsal bone & & & 4.6 \\
Proximal & $1.11 \pm 0.39$ & $0.96 \pm 0.32$ & $0.73(0.39-0.88)$ & 4.6 \\
Shaft & $1.68 \pm 0.46$ & $1.79 \pm 0.46$ & $0.92(0.74-0.97)$ & 2.8 \\
Distal & $0.55 \pm 0.21$ & $0.56 \pm 0.20$ & $0.90(0.81-0.95)$ & 4.2 \\
Total & $3.34 \pm 0.89$ & $3.31 \pm 0.87$ & $0.95(0.89-0.97)$ & 3.9 \\
\hline Intra class Correlation Coefficient (ICC), Standard Error of Measure (SEM)
\end{tabular}

Intra class Correlation Coefficient (ICC), Standard Error of Measure (SEM) 
Table 6 BMC Scan-Rescan reliability

\begin{tabular}{|c|c|c|c|c|}
\hline Variable & $\begin{array}{l}\text { Mean }{ }_{D_{1}} \\
\left(\mathrm{~g} / \mathrm{cm}^{2}\right)\end{array}$ & $\begin{array}{l}\text { Mean }_{\mathrm{D} 2} \\
\left(\mathrm{~g} / \mathrm{cm}^{2}\right)\end{array}$ & ICC (95\% Cl) & SEM (\%) \\
\hline \multicolumn{5}{|c|}{ Fifth Metatarsal bone } \\
\hline Proximal & $1.87 \pm 0.63$ & $1.94 \pm 0.73$ & $0.89(0.78-0.95)$ & 12.8 \\
\hline Shaft & $1.32 \pm 0.40$ & $1.36 \pm 0.40$ & $0.83(0.66-0.91)$ & 12.6 \\
\hline Distal & $0.33 \pm 0.11$ & $0.34 \pm 0.12$ & $0.66(0.39-0.83)$ & 21.6 \\
\hline Total & $3.53 \pm 1.08$ & $3.63 \pm 1.20$ & $0.89(0.79-0.95)$ & 11.0 \\
\hline \multicolumn{5}{|c|}{ Second Metatarsal bone } \\
\hline Proximal & $1.11 \pm 0.39$ & $1.08 \pm 0.36$ & $0.66(0.40-0.82)$ & 20.8 \\
\hline Shaft & $1.68 \pm 0.46$ & $1.65 \pm 0.45$ & $0.87(0.75-0.94)$ & 10.0 \\
\hline Distal & $0.55 \pm 0.21$ & $0.54 \pm 0.21$ & $0.82(0.65-0.91)$ & 16.6 \\
\hline Total & $3.34 \pm 0.89$ & $3.27 \pm 0.90$ & $0.88(0.77-0.94)$ & 9.4 \\
\hline
\end{tabular}

Intra class Correlation Coefficient (ICC), Standard Error of Measure (SEM)

group [11]. However, this relationship was not found in BSI's of the metatarsals [11]. While increased BMD seems to be strongly related to fracture risk, whole body BMD may not be related to metatarsal BMD measurements in athletes and thus, may not be a good indicator of metatarsal fracture risk [17]. It may be that DXA BMD measurements of the metatarsals will give a better indication of injury risk than whole body BMD measurements. Furthermore, BMD measurements of the proximal metatarsals may yield better insight into fractures of this site.

Knowledge of an individual's metatarsal bone mineral density distribution could be useful in designing interventions to prevent these injuries. Bone constantly remodels itself to adapt to the loads that are being applied to it [18]. Through training, it is possible to increase the bone mineral content of specific bones; however, it is uncertain to what limit this remodeling takes place [19]. In a comparison of 3 groups of runners, lowdistance $(5-30 \mathrm{~km} / \mathrm{wk})$ middle-distance $(30-50 \mathrm{~km} / \mathrm{wk})$

Table 7 Area Intra-rater reliability

\begin{tabular}{|c|c|c|c|c|}
\hline Variable & $\begin{array}{l}\text { Mean }{ }_{A 1} \\
\left(\mathrm{~g} / \mathrm{cm}^{2}\right)\end{array}$ & $\begin{array}{l}\text { Mean }_{A 2} \\
\left(\mathrm{~g} / \mathrm{cm}^{2}\right)\end{array}$ & ICC $(95 \% \mathrm{Cl})$ & SEM (\%) \\
\hline \multicolumn{5}{|c|}{ Fifth Metatarsal bone } \\
\hline Proximal & $3.7 \pm 0.53$ & $3.57 \pm 0.54$ & $0.80(0.60-0.90)$ & 6.7 \\
\hline Shaft & $2.9 \pm 0.51$ & $2.96 \pm 0.50$ & $0.93(0.85-0.97)$ & 4.7 \\
\hline Distal & $1.29 \pm 0.21$ & $1.22 \pm 0.18$ & $0.72(0.39-0.87)$ & 9.0 \\
\hline Total & $7.89 \pm 1.01$ & $7.74 \pm 0.96$ & $0.92(0.83-0.96)$ & 3.6 \\
\hline \multicolumn{5}{|c|}{ Second Metatarsal bone } \\
\hline Proximal & $0.65 \pm 0.13$ & $0.64 \pm 0.14$ & $0.95(0.90-0.98)$ & 4.9 \\
\hline Shaft & $0.51 \pm 0.09$ & $0.51 \pm 0.09$ & $0.98(0.96-0.99)$ & 2.4 \\
\hline Distal & $0.31 \pm 0.06$ & $0.31 \pm 0.06$ & $0.97(0.93-0.98)$ & 3.9 \\
\hline Total & $0.49 \pm 0.08$ & $0.48 \pm 0.09$ & $0.97(0.92-0.98)$ & 3.4 \\
\hline
\end{tabular}

Intra class Correlation Coefficient (ICC), Standard Error of Measure (SEM)
Table 8 Area Inter-rater reliability

\begin{tabular}{lllll}
\hline Variable & $\begin{array}{c}\text { Mean } \\
\left(\mathrm{g} / \mathrm{cm}^{2}\right)\end{array}$ & $\begin{array}{l}\text { Mean }_{\mathrm{A2}} \\
\left(\mathrm{g} / \mathrm{cm}^{2}\right)\end{array}$ & ICC (95\% Cl) & SEM (\%) \\
\hline Fifth Metatarsal bone & & & \\
Proximal & $3.7 \pm 0.53$ & $3.52 \pm 0.50$ & $0.77(0.51-0.90)$ & 7.0 \\
Shaft & $2.9 \pm 0.51$ & $3.38 \pm 0.41$ & $0.52(-0.1-0.82)$ & 11.3 \\
Distal & $1.29 \pm 0.21$ & $1.47 \pm 0.26$ & $0.54(0.02-0.79)$ & 12.9 \\
Total & $7.89 \pm 1.01$ & $8.38 \pm 0.90$ & $0.78(0.18-0.92)$ & 5.9 \\
Second Metatarsal bone & & & \\
Proximal & $1.71 \pm 0.35$ & $1.49 \pm 0.29$ & $0.45(0.06-0.71)$ & 16.5 \\
Shaft & $3.28 \pm 0.54$ & $3.57 \pm 0.49$ & $0.75(0.11-0.91)$ & 8.0 \\
Distal & $1.73 \pm 0.40$ & $1.85 \pm 0.36$ & $0.67(0.41-0.83)$ & 12.7 \\
Total & $6.72 \pm 0.86$ & $6.90 \pm 0.87$ & $0.86(0.71-0.93)$ & 4.8 \\
\hline
\end{tabular}

Intra class Correlation Coefficient (ICC), Standard Error of Measure (SEM)

and long-distance (50-100 km/wk), bone mineral density was higher in the middle and long distance runners compared to low-distance runners and similar between middle and long-distance runners [19]. Thus, whilst chronic loading is vital to the bone remodeling process, at some point, the amount of chronic loading will not produce any greater gains in bone mineral density. This limit may be important in identifying fracture risk.

\section{Conclusions}

In summary, a reliable technique for assessing bone mineral density of shaft and sub-regions of the shaft of the second and fifth metatarsals was performed. This could be used in laboratories to screen for individuals who may be at risk for metatarsal fractures. Furthermore, the reliability of the segmented ROI's may provide additional insight into fracture risk at the metatarsals.

Table 9 Area Scan-Rescan reliability

\begin{tabular}{ccccc}
\hline Variable & $\begin{array}{c}\text { Mean } 1 \\
\left(\mathrm{~g} / \mathrm{Cm}^{2}\right)\end{array}$ & $\begin{array}{l}\text { Mean } 2 \\
\left(\mathrm{~g} / \mathrm{Cm}^{2}\right)\end{array}$ & ICC $(95 \% \mathrm{Cl})$ & SEM (\%) \\
\hline Fifth Metatarsal bone & & & \\
Proximal & $3.7 \pm 0.53$ & $3.72 \pm 0.55$ & $0.83(0.68-0.92)$ & 6.9 \\
Shaft & $2.9 \pm 0.51$ & $2.94 \pm 0.41$ & $0.73(0.51-0.87)$ & 9.0 \\
Distal & $1.29 \pm 0.21$ & $1.28 \pm 0.27$ & $0.57(0.26-0.77)$ & 13.9 \\
Total & $7.89 \pm 1.01$ & $7.94 \pm 1.00$ & $0.89(0.77-0.94)$ & 4.3 \\
Second Metatarsal bone & & & 20.4 \\
Proximal & $1.71 \pm 0.35$ & $1.68 \pm 0.42$ & $0.32(-0.05-0.61)$ & 16.0 \\
Shaft & $1.73 \pm 0.40$ & $1.77 \pm 0.43$ & $0.58(0.28-0.78)$ & 7.3 \\
Distal & $3.28 \pm 0.54$ & $3.24 \pm 0.59$ & $0.83(0.68-0.92)$ & 6.2 \\
Total & $6.72 \pm 0.86$ & $6.69 \pm 1.03$ & $0.84(0.69-0.92)$ & 6 \\
\hline
\end{tabular}

Intra class Correlation Coefficient (ICC), Standard Error of Measure (SEM) 


\section{Abbreviations}

2MT: Second metatarsal; 5MT: Fifth metatarsal; BMC: Bone mineral content; BMD: Bone mineral density; BSI: Bone stress injury; DXA: Dual x-ray absorptiometry; ICC: Intra class correlation coefficient; qCT: Quantitative computed tomography; ROI: Region of interest; SEM: Standard error of measurement

\section{Acknowledgements}

Not applicable.

\section{Funding}

Not applicable.

\section{Availability of data and materials}

The datasets generated and/or analyzed during the current study are not publicly available due to individual privacy concerns but are available from the corresponding author on reasonable request.

\section{Authors' contributions}

NSP assisted in creating the scan protocol, analyzed the scans, and was a major contributor in writing the manuscript. JMS assisted in creating the scan protocol, was a large contributor to the study design, and assisted with writing the manuscript. AN contributed to the study design and assisted with writing the manuscript. MSB assisted in creating the scan protocol, analyzed the scans, and assisted with writing the manuscript. DRS contributed to the study design, assisted in creating the scan protocol, and assisted with writing the manuscript. JBT contributed to the study design and assisted with writing the manuscript. KRF contributed to the study design, assisted in creating the scan protocol, assisted with data analyses, and assisted with writing the manuscript. All authors read and approved the final manuscript.

\section{Authors' information}

Not applicable

\section{Ethics approval and consent to participate}

Participants signed a consent form to participate in the research study that was approved by High Point University IRB.

\section{Consent for publication}

Not applicable

\section{Competing interests}

The authors declare that they have no competing interests.

\section{Publisher's Note}

Springer Nature remains neutral with regard to jurisdictional claims in published maps and institutional affiliations.

\section{Author details}

${ }^{1}$ Department of Physical Therapy, High Point University, High Point, NC, USA. ${ }^{2}$ Department of Athletic Training, High Point University, High Point, NC, USA. ${ }^{3}$ Washington University School of Medicine, St. Louis, MO, USA.

Received: 14 July 2017 Accepted: 15 November 2017

Published online: 28 November 2017

\section{References}

1. Iwamoto J, Sato Y, Takeda T, Matsumoto H. Analysis of stress fractures in athletes based on our clinical experience. World J Orthop. 2011;2:7-12

2. Zwitser EW, Breederveld RS. Fractures of the fifth metatarsal; diagnosis and treatment. Injury. 2010;41:555-62

3. Torg JS, Balduini FC, Zelko RR, Pavlov H, Peff TC, Das M. Fractures of the base of the fifth metatarsal distal to the tuberosity. Classification and guidelines for non-surgical and surgical management. J Bone Joint Surg Am. 1984:66:209-14.

4. Bowes J, Buckley R. Fifth metatarsal fractures and current treatment. World J Orthop. 2016:7:793-800.

5. Chuckpaiwong B, Cook C, Pietrobon R, Nunley JA. Second metatarsal stress fracture in sport: comparative risk factors between proximal and nonproximal locations. Br J Sports Med. 2007:41:510-4.
6. Jain N, Murray D, Kemp S, Calder J. Frequency and trends in foot and ankle injuries within an English premier league football Club using a new impact factor of injury to identify a focus for injury prevention. Foot Ankle Surg. 2014:20:237-40.

7. Cakir H, Van Vliet-Koppert ST, Van Lieshout EM, De Vries MR, Van Der Elst M, Schepers T. Demographics and outcome of metatarsal fractures. Arch Orthop Trauma Surg. 2011;131:241-5.

8. Dameron TB Jr. Fractures of the proximal fifth metatarsal: selecting the best treatment option. J Am Acad Orthop Surg. 1995;3:110-4.

9. Bakkum AJ, Janssen TW, Rolf MP, Roos JC, Burcksen J, Knol DL, de Groot S. A reliable method for measuring proximal tibia and dista femur bone mineral density using dual-energy X-ray absorptiometry. Med Eng Phys. 2014;36:387-90.

10. Geusens P, De Winter L, Quaden D, Vanhoof J, Vosse D, van den Bergh J, Somers $\mathrm{V}$. The prevalence of vertebral fractures in spondyloarthritis: relation to disease characteristics, bone mineral density, syndesmophytes and history of back pain and trauma. Arthritis Res Ther. 2015:17:294.

11. Tenforde AS, Carlson JL, Chang A, Sainani KL, Shultz R, Kim JH, Cutti P, Golden NH, Fredericson M. Association of the Female Athlete Triad Risk Assessment Stratification to the development of bone stress injuries in collegiate athletes. Am J Sports Med. 2017;45:302-10.

12. Fuller JT, Archer J, Buckley JD, Tsiros MD, Thewlis D. The reliability of dualenergy $\mathrm{X}$-ray absorptiometry measurements of bone mineral density in the metatarsals. Skelet Radiol. 2016:45:135-40.

13. Baim S, Wilson CR, Lewiecki EM, Luckey MM, Downs RW Jr., Lentle BC. Precision assessment and radiation safety for dual-energy $\mathrm{X}$-ray absorptiometry: position paper of the International Society for Clinical Densitometry. J Clin Densitom. 2005;8:371-8.

14. Bohnert KL, Gutekunst DJ, Hildebolt CF, Sinacore DR. Dual-energy X-ray absorptiometry of human metatarsals: precision, least significant change and association to ex vivo fracture force. Foot (Edinb). 2013;23:63-9.

15. Weir JP. Quantifying test-retest reliability using the intraclass correlation coefficient and the SEM. J Strength Cond Res. 2005:19:231-40.

16. Commean PK, Ju T, Liu L, Sinacore DR, Hastings MK, Mueller MJ. Tarsal and metatarsal bone mineral density measurement using volumetric quantitative computed tomography. J Digit Imaging. 2009;22:492-502.

17. Courtney AC, Davis BL, Manning T, Kambic HE. Effects of age, density, and geometry on the bending strength of human metatarsals. Foot Ankle Int. 1997;18:216-21

18. Wang J, Tan S, Cao L. Site-specific relationship between bone mineral density and muscle strength or endurance in elderly men. Asian J Exerc Sport Sci. 2014;11:36-45.

19. Deriaz O, Najafi B, Ballabeni P, Crettenand A, Gobelet C, Aminian K, Rizzoli R, Gremion G. Proximal tibia volumetric bone mineral density is correlated to the magnitude of local acceleration in male long-distance runners. J Appl Physiol (1985). 2010;108:852-7.

\section{Submit your next manuscript to BioMed Central and we will help you at every step:}

- We accept pre-submission inquiries

- Our selector tool helps you to find the most relevant journal

- We provide round the clock customer support

- Convenient online submission

- Thorough peer review

- Inclusion in PubMed and all major indexing services

- Maximum visibility for your research

Submit your manuscript at www.biomedcentral.com/submit
BioMed Central 